\title{
A Pragma-Dialectical Study of Pragmatic Argumentation in Academic Argument
}

\author{
Jingjing Ma *, Hong Chen \\ School of Foreign Languages, Jiangsu University, Zhenjiang, China \\ Email:1048288754@qq.com
}

Keywords: academic argumentation; Pragma-Dialetics; applied linguistics; prototypical pattern

Abstract: Academic argumentation refers to a concrete form of theoretical thinking embodied in academic works. It is a process of exposition of scientific materials to illustrate academic issues and proves that academic opinions have a true value. Academic argumentation is also an inference method and form exploited by countless preconditions to withdraw conclusions. In academic papers, authors are likely to use practical arguments to convince their target audiences by highlighting the positive or negative results that a particular standpoint has. Current research on pragmatic argumentation in institutional contexts mainly concentrates on the legal and political domains. Few researchers pay enough attention to pragmatic argumentation used in academic argument, let alone formulate proper soundness criteria for evaluating its reasonableness. Given all this, within the framework of Pragma-dialectics, this paper analyzes and evaluates the pragmatic argumentation typically used in the paper How Applied Linguistics is the same as other science in the Applied Linguistics. The general goal of this research is to reveal how pragmatic argumentation is used in academic argument. Based on the institutional preconditions of academic argument and the general pragma-dialectical soundness criteria for pragmatic argumentation, this study puts forward specific criteria for evaluating pragmatic argumentation in case of academic argument. This paper aims to evaluate the use of pragmatic argumentation in How Applied Linguistics is the same as other science and finds that academic argument may sometimes fail to keep the balance between reasonableness and effectiveness and consequently commits derailed fallacies, when they overwhelmingly pursue the persuasive effects of pragmatic argumentation and its extensions. This research is of both theoretical and practical significance. In theory, this study develops a pragma-dialectical research framework for academic argument, thus expanding the research perspectives and methods of academic argument. Meanwhile, the scope of institutional contexts of Pragma-dialectics is extended by studying academic argument. In practice, this study enlightens academic researchers who are responsible for international communication on how to use pragmatic argumentation both reasonably and effectively. More importantly, it provides a crucial analytic tool for readers to interpret academic argument appropriately and improve their critical thinking abilities. 


\section{Introduction}

\subsection{Research Background}

Academic dissertation is the dissertation that expresses the scientific research results after making a comparative and systematic research and discussion on the problems in a certain field. Academic papers are basically academic, scientific, creative, and theoretical. The theoretical nature of academic papers is not only reflected in the theoretical nature of the mind and the author's theoretical cultivation, but also reflected in the argumentative nature of the expression. The theoretical characteristic stems from the process of argument and reasoning. Argument plays an essential role in academic writing, thus it is of great significance to analyze academic argument. The academic contextualized argument can also be defined as the structure supporting the particular writer's ideas, and supported by reasoning and referencing that are acceptable and persuasive in one particular academic realm. To conclude, Argument plays an essential role in academic writing, thus it is of great significance to analyze and evaluate academic argument

\subsection{Aim and Significance}

This paper aims to make clear how pragmatic argumentation is used in academic argument and what the proper criteria is for evaluating its use. It seeks to accomplish the following three research aims:

(1) do a tentative exploration of pragmatic argumentation used in the argument of academic essay;

(2) roughly introduce the theory of Pragma-Dialects and classify complex pragmatic argumentation based on prototypical argument patterns;

(3) evaluate the reasonableness of the use of pragmatic argumentation in the paper How Applied Linguistics is same as other science

The outcomes of the current research will also have practical implications. First, this paper integrates academic argument with pragmatic argumentation, providing a new research perspective. Second, the application of pragmatic argumentation is expanded and studies on it are enriched, too. Third, it provides a new set of rule to analyze and evaluate academic argument.

\subsection{Organization of the Thesis}

This paper is made up of five chapters as follows:

The first chapter introduces an argumentative approach to academic argument and provides a brief account of the research background, aims and significance. It also introduces the structure of this paper and the content of each chapter.

The second chapter introduces previous studies on academic argument and Pragma-Dialects.

The third chapter demonstrates the research design, including the theoretical assumptions, research questions and research methods.

The fourth chapter firstly discusses argumentative characterization of academic argument. This section deals with the argumentative characterization and institutional preconditions of pragmatic argumentation used in academic argument. The second section is prototypical argument schemes of pragmatic argumentation. It introduces the prototypical pragmatic argumentation. The third section is evaluation of prototypical pramagtic argument. Pragmatic argumentation will be dug out from the paper How Applied Linguistics is same as other Science and be analyzed from four stages. Besides, the effectiveness of them will be judged by different aspects. In the fourth section, criteria is provided to evaluate pragmatic argumentation in academic argument. 
The fifth chapter summarizes the whole paper and points out the limitations of this paper and further research of this field.

\section{Literature Review}

\subsection{Studies on academic argument}

The most basic logical method of academic argumentation is to observe the general laws of formal logic. Lu (2006) studies how to conduct academic argument and concludes that Academic argumentation is the concrete application of logical knowledge, and it is a concrete manifestation of logical thinking ability. Xia(2017) concludes that the academic contextualized argument may be defined as the structure supporting the particular writer's ideas, and supported by reasoning and referencing that are acceptable and convincing in one specific academic field. J. Wagemans (2015) proposes the basic pattern of academic argument from the practice of science and philosophical.

\subsection{Studies on Pragma-dialects}

The pragma-dialectical theory of argumentation originated from the University of Amsterdam by Frans H. van Emerson and Rob Grootendorst in the 1970s and developed over the following four decades. As the name of this theory indicates, the integration of dialectical and pragmatic insights is the distinctive characteristic of pragma-dialects.

The various contributions to pragma-dialects as a typical argumentative pattern in certain communicative activity types belonging to the political domain, the legal domain, the medical domain, and the academic domain, have been made recently. In the context parliamentary debate, Garssen (2015), on the basis of the basic scheme of pragmatic argumentation, puts forward the pragmatic problem-solving argumentation. Focusing on the case of European parliamentary committees of inquiry, Andone (2015) pays attention to the argumentative patterns combining pragmatic argumentation in which a recommendation is made with an argument invoking the majority will. Feteris (2015) explores the function and implementation of pragmatic argumentation in prototypical argumentative patterns in legal justification. In the medical domain, Henkemans (2015) identifies pragmatic argumentation and presents various types of extensions of this basic argumentative pattern which are often used by the advertisers as the strategic choices to promote their products. And in the academic domain, Wagemans (2015) makes clear the important role of pragmatic argumentation played in justifying scientific explanations.

To summarize, there is much room for us to explore the practical applications of pragmatic argumentation like in the academic realm.

\subsection{Summary}

Based on the previous research mentioned above, we can assert that though some studies have been done on the academic argument field, mainly from the perspective of logistics, linguistics and pragmatics, the majority of them do not pay attention to the argumentative feature of it, especially those practical argumentative patterns which often occur in them, such as pragmatic argumentation as a main argument that are typical of argumentative discourse in the political, the legal, the medical, and the academic domain. This paper will identify and analyze pragmatic argumentation used in the paper How Applied Linguistics is same as other science. What's more, four stages of pragmatic argumentation will be given according to their argument schemes. At last, the effectiveness of this paper will be evaluated from the perspective of pragmatic argumentation. 


\section{The Theoretical Framework of Pragma-Dialects}

This chapter deals with the research design, including the theoretical assumptions, research questions and research methods.

\subsection{Theoretical Assumptions}

\subsubsection{The definition of argumentation}

In pragma-dialectics, argumentation is defined as "a communicative and interactional (speech) act complex aimed at resolving a difference of opinion before a reasonable judge by advancing a constellation of reasons the arguer can be held accountable for as justifying the accept ability of the standpoint(s) at issue" (van Eemeren, 2010, p. 29). Within the structure of Pragma-dialectics, argumentation is researched both from a communicative perspective relating to language philosophy, speech act theory and discourse analysis, and from a critical perspective relating to critical reasonableness and dialogue logic. The dialectical dimension is inspired by normative insights from "critical reasonableness" and formal dialectics, the pragmatic dimension by descriptive insights from speech act theory, Gricean language philosophy and discourse analysis.

\subsubsection{Ideal model of a critical discussion}

The ideal model of a critical discussion intends to resolve a difference of opinion by arguing whether the standpoint at issues should be accepted or not. It takes place between the protagonist who defends a certain standpoint and the antagonist who doubts or challenges the proposed standpoint. To allow for the systematic integration of the pragmatic and dialectical dimensions in the study of argumentation, the pragma-dialectical theory uses four meta-theoretical principles as its point of departure: functionalization, socialization, externalization and dialectification. Functionalization is achieved by treating discourse as a purposive act. Socialization is achieved by extending the speech act perspective to the level of interaction. Externalization is achieved by capturing the propositional and interactional commitments created by the speech acts performed. And dialectification is achieved by regimenting the exchange of speech acts to an ideal model of a critical discussion.

In an ideal model of critical discussion, argumentative discourse is classfied analytically into four stages: the confrontation stage, the opening stage, the argumentation stage and the concluding stage. In the confrontation stage, the parties make clear that they have a difference of opinion and identify the types of difference of opinion. In the opening stage, they decide to resolve this difference of opinion. The interlocutors determine their points of departure: they agree on the rules of the discussion and establish which propositions they can use in their argumentation. In the argumentation stage, the protagonist defends their standpoint by advancing arguments to eliminate the antagonist's doubt or counter the antagonist's objections. In the concluding stage, the discussion parties evaluate to what extent their initial difference of opinion has been resolved and in whose favor. The model also defines the nature and distribution of the speech acts that play a constructive part in the various stages of the resolution process.

\subsubsection{Strategic maneuvering}

Individuals involved in real-world argumentative discourse are always faced with the "argumentative predicament" that every argumentative move taking aim at effectiveness needs to go together with maintaining rationality. In argumentative reality, individuals are inclined to resolve a difference of opinion reasonably by abide by certain critical standards of reasonableness. However, 
in the mean time, some people are possibly primarily interested in resolving the difference of opinion efficiently in their favor.

Recently, the pragma-dialectical theory of argumentation has incorporated insights from rhetoric into the analysis of argumentative discussion. Parties involved in a difference of opinion "maneuver strategically" to simultaneously realize their dialectical and their rhetorical aims. In other words, the parties in an argumentative discussion attempt to be persuasive (have their standpoint accepted) while observing the critical standards for argumentative discourse. In each of the critical discussion stages there is a rhetorical goal that corresponds with the dialectical goal and interlocutors can make use of three analytical aspects to balance effectiveness and reasonableness: making an opportune selection from the topical potential available at the stage concerned, approaching the audience efficiently and carefully exploiting presentational means. These three aspects correspond with some focal points of rhetorical study - topics, audience adaptation and presentational devices - so that insights acquired in rhetoric are brought to bear in explaining how rhetorical and dialectical considerations play a part in the various ways of strategic maneuvering.

\subsubsection{Pragmatic Argumentation}

Pragmatic argumentation is defined by a series of argumentative moves aiming at solving certain differences of opinion and it is defended by explaining or illustrating the desirable or undesirable results resulting from the actions the arguers support or oppose (Frans van Eemeren, 2015). Also, pragmatic argumentation has two variants: positive variant and negative variant.

Pragmatic argumentation that is used to support a positive standpoint is generalized as "Action $\mathrm{X}$ should be carried out". On contrast, the pragmatic argumentation applied to a negative standpoint can be represented as "Action X should not be carried out". The pragma-dialectical definition of the argument scheme of pragmatic argumentation in positive version is as follows (van Eemeren, 2015): 1 Standpoint Action $X$ should be carried out 1.1 Because Action $X$ will lead to positive result $\mathrm{Y}\left(1.1^{\prime}\right) 1$ And (Actions of type $\mathrm{X}$ [such as $\mathrm{X}$ ] that lead to positive results of type $\mathrm{Y}$ [such as $\mathrm{Y}$ ] must be carried out). Pragmatic argumentation will be evaluated in accordance with the critical questions pertinent to the argument scheme of causal argumentation. This means that the following critical questions can be raised (van Eemeren, 2015): (a) Do actions of type X lead to results of type Y? (b) Is result Y really positive (i.e., desirable)/negative (i.e., undesirable)? (c) Does action X not have any major negative (i.e., undesirable)/positive (i.e., desirable) side-effects?

\subsection{Research Questions}

Based on the theoretical basis of pragmatic argumentology, this study begins with path of argumentative discourse analysis and analyzes the "argumentability" of academic argument, and specifically discusses the following issues:

How pragmatic argumentation used in academic argument?

What is the basic model of pragmatic argumentation used in academic argument in How applied linguistics is same as other science? What are their strategic functions?

How to evaluate pragmatic argumentation used reasonably in academic argument?

Did How applied linguistics is same as other science keep to a balance between dialectical reasonableness and rhetorical effectiveness when using pragmatic argumentation?

\subsection{Research Method}

This study is based on the basic theories and methods of pragmatic argumentation, constructing a critical analysis framework based on "discourse structure analysis-evaluation of pragmatic 
effects-rational criticism of argumentation". This analytical framework not only takes pragmatic, argumentative, rhetorical, and logical characteristics of the argumentative of the argumentative discourse, and can fully reflect the "argumentative" dimensions of critical thinking cognitive process in academic argument.

\subsubsection{Reconstructing argumentative discourse}

First of all, the critical analysis refers to the identification, reconstruction, and reproduction of the different argumentative discourse structures of both parties in the field of communicative discourse of each communicative field. Based on this, it analyzes and compares the six core components of the discourse of each communicative field. (including disagreements, starting points, positions, implicit prerequisites, diagram form and conclusions) and linguistic features. Linguistic features analysis is not limited to microscopic descriptions (including speech and semantics) of single words and sentence grammatical structures under the category of linguistics. Syntax) should also involve more complex and advanced levels such as textual cohesion and intertextuality.

\subsubsection{Analyzing pragmatic argumentation in academic argument}

When trying to analyze pragmatic argumentation in academic essays, it is required to verdict whether the standpoint is prescriptive or not. The judgment of the type of standpoint is the first step to make a pragma-dialectical analysis of pragmatic argumentation. Pragmatic argumentation belongs to the sub-type of causal argumentation and it works as a specific type of strategic maneuver in argumentation discourse with the goal to convince target readers effectively. To permit a more sophisticated analysis of every strategic maneuver, it is essential to take the three aspects of strategic maneuvering into consideration: topical potential, audience demand, and presentational device. According to van Eemeren (2016), pragmatic argumentation has its own argument scheme consisting of a standpoint, an explicit premise and an unexpressed premise. Concerning the specific institutional context of academic argument, the 29 institutional conventions impose certain constraints on the using of pragmatic argumentation. Moreover, the institutional aims that commentators attempt to realize stimulate the use of its extensions. In this view, both the basic argument scheme of pragmatic argumentation and the institutional contexts of academic argument should be taken seriously as the analytic tool to analyze the pragmatic argumentation used in academic argument.

\subsubsection{Evaluating pragmatic argumentation in academic argument}

To evaluate the reasonableness of pragmatic argumentation used in academic argument, soundness criteria should be explored to evaluate whether the use is reasonable or fallacious.

Critical evaluation refers to the fact that the subject manipulates argument rhetoric in discourse communication to obtain linguistic pragmatic effects. The two sides of the communication maintain their own "attitudes" more effectively within a reasonable range, and each implements "trick" manipulation. The recognition of attitudes and skills must be completed through critical evaluation. This includes assessing the rhetorical elements of discourse and its linguistic features from the perspectives of rationality (according to the criterion of reasonableness) and rhetorical validity (effectively persuading the other party or other audience), and then summarizing and contrasting the high frequencies in the process of argumentation.

Considering the argumentative reality, soundness criteria stresses the institutional constraints that academic argument as a special argumentative activity type imposes on the specific use of pragmatic argumentation.

This study uses the paper How applied linguistics is same as other science as an example and 
explain how to explore the cognitive processing of critical thinking through the analysis, evaluation, and critical path of discourse.

\section{Results and Discussions}

The paper How Applied Linguistics is same as other science, which was published in the journal of Applied Linguistics in 1997. Applied Linguistics is a peer-reviewed academic journal in the field of applied linguistics established in 1980 and published by Oxford University Press. The journal publishes both research papers and conceptual articles in all aspects of applied linguistics, such as lexicography, corpus linguistics, multilingualism, discourse analysis, and language education, aiming at promoting discussion among researchers in different fields. It features a "Forum" section, introduced in 2001, intended for short contributions, such as responses to articles and notices about current research. Due to the length of paper, the following paragraphs are selected to analyze:

(1)At this point it should be clear that the conflation of Widdowson-Brumfit is inappropriate. (2) Anyone reading the work of either of us over the past two decades will immediately see that Widdowson's work is much more overtly linguistic than mine is. (3)His literary work (Widdowson 1992) depends substantially on detailed analysis of alternative versions of texts and the analysis of these readings; his work on discourse, on languages for specific purposes, or his recent critique of critical approaches (Widdowson 1995) depend on linguistic theory and linguistic analysis to a far greater extent than most of my own writings do.(4) Whatever similarities there may sometimes be in our justifications of the applied linguistic enterprise, our practices are substantially divergent.

In the sample paragraph, confronting with the different opinions, the author generally shows his negative attitude by indicating that opposing certain standpoint would prevent a negative result. In order to support this argument, the author commonly applies pragmatic argumentation to defend his standpoint. The basic pragmatic argumentation can therefore be summarized as:

Issue $\mathrm{X}$ should not be supported

a. Issue $\mathrm{X}$ will cause damage $\mathrm{Y}$

b. Issue of type $\mathrm{X}$ [such as $\mathrm{X}$ ] that causes damage of type $\mathrm{Y}$ [such as $\mathrm{Y}$ ] should not be supported

In order to illustrate how the prototypical pragmatic argumentation works in real context, analysis of example reveals the strategic choice made by the author in convincing the readers.

In the sample text, sentence (1) clearly states the standpoint that "the conflation of Widdowson-Brumfit is inappropriate". Referring to the relevant background of applied linguistics, it is easy to understand the suppressed premises:(1) The viewpoints of Widdowson is different from the viewpoints of Brumfit.(2) Alhough they may share some similar characteristics, their theories are radically different.(3) The combination of Widdowson's viewpoints and Brumift's viewpoints is illogical. H. G. Widdowson is an internationally acclaimed authority in applied linguistics and language teaching. He is one of the main advocators, promoters and preachers of communicative approach. Christopher Brumfit is the author of this paper. He asserts that it is not appropriate to combine Widdowson's viewpoints with his own's. It can be inferred from this part of the context that the author tries to eliminate the disagreement that whether it is appropriate to combine Widdowson's standpoints with his own viewpoints. In this part the author clearly expressed the disagreements and denial standpoints.

In order to maintain and demonstrate this standpoint of denial, Communicators must necessarily choose the starting point of the argument that best suits their interests at the opening stage, that is, the facts or value judgment that both sides consent or default explicitly. This strategic fact or value selection actually reflects the intentionality of the elite groups which the communicators represent. 
In this paragraph, the author choices sentence (2) and sentence (3) as the two starting points to maintain his standpoints. It can be concluded from this two sentences that Widdowson's work is distinctively more linguistic. Also, his critical approaches depend on linguistic theory. It is intended to further prove his standpoint that the conflation of Widdowson-Brumift is not appropriate. In order to prove that "the conflation of Widdowson-Brumift is inappropriate", the author conducts the argument in each sentence of this paragraph. Among them, the argument of sentence (1) mainly argues that it is not appropriate to combine Widdowson's opinions with his own viewpoints. The structure of argument can be expressed as:

Argument 1 at this point it should be clear that the conflation of Widdowson-Brumfit is inappropriate.

a. The viewpoints of Widdowson are different from the viewpoints of Brumfit.

b. Although they may share some similar characteristics, their theories are radically different.

c. The combination of Widdowson's viewpoints and Brumift's viewpoints are unreasonable.

Reconstruction of discourse structure displays that the author uses argument a and b to justify "The combination of Widdowson's viewpoints and Brumift's viewpoints is illogical". In other words, if $\mathrm{a}$ and $\mathrm{b}$ are both valid, and there is indeed a representative relationship between "The viewpoints of Widdowson is different from the viewpoints of Brumfit" and "their theories are radically different", then argument 1 holds. This argument strategy is referred to as "argumentation based on a symptomatic relation" by Emerson in 2002. In sentence (2), the author justify a by providing facts(Widdowson's work, much more overtly linguistic). This argument ascribes the irrationality of the conflation of Widdowson-Brumfit to the divergence of their theories. Because Widdowson's work is much more linguistic(overtly linguistic). In the meantime, the author also presupposes a debate structure for later texts: Widdowson's literary work and his work on discourse are closely related to linguistic field, which can be demonstrated and justified through the following sentence:

Argument 2 Anyone reading the work of either of us over the past two decades will immediately see that Widdowson's work is much more overtly linguistic than mine is.

a. For readers who have a little understanding of our works, it is obvious that our works are different.

b. Almost Any individual can distinguish that our works are different.

c. Widdowson's work is more linguistic than mine.

d.It is quite easy to distinguish that Widdowson studies more in linguistic field.

Argument 2 contains two argument schemes. $a$ and $b$ constitute reasons together, and form argumentation based on a causal relation with argument 2, a, b and c form Symptomatic relational argument. The two schemes work together, constituting mixed-type complex argument structure. This structure is illustrated in figure:

The analysis and reconstruction of the holistic discourse structure can not only clearly demonstrate the author's argumentation skills in rationalizing his own standpoint, but also help the reader to effectively identify the author's attitude and facilitate the judgment of the rationality and effectiveness of the discourse.

According to the reconstruction of this case, concerning the ten rules of pragmatic argumentation and the background of applied linguistics, we come to a conclusion that there are violations in the argument scheme rule (rule seven) and validity rules (rule eight). For instance, both argument 1 and argument 2 show some irrationality. 


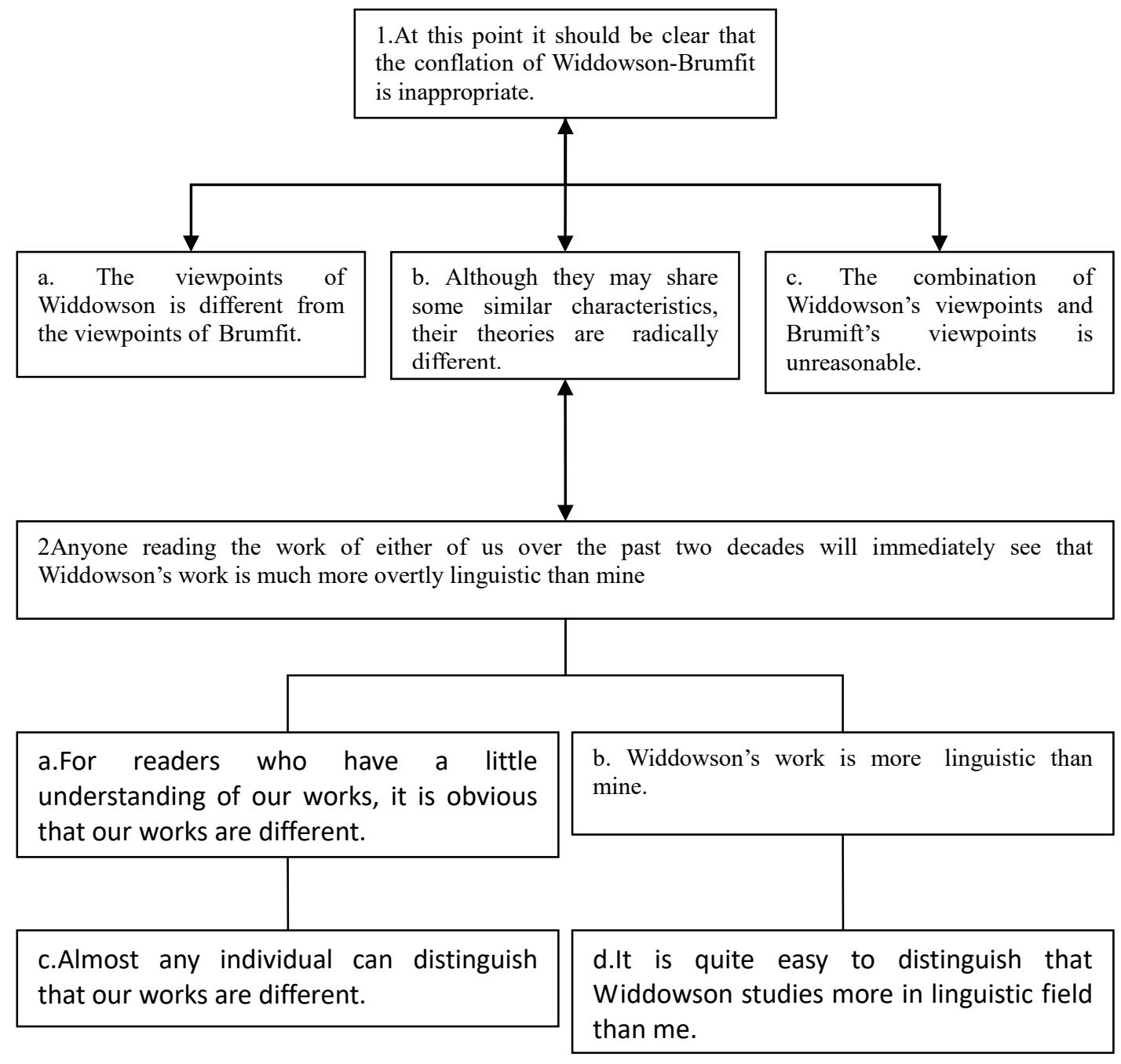

Figure 1 The Argument and structure diagram

Firstly, the author violates the rule of argument scheme. The so-called argument scheme refers to the abstract reasoning relationship in the argument structure that can change the acceptability of the (large and small) premise to the acceptability of the conclusion. Argumentation Scheme rules require that the arguer correctly use the appropriate argument scheme to maintain his standpoint. In pragma-dialects, the reasoning structure of the symptomatic argument schema can be illustrated as:

$$
\text { Conclusion } \quad \mathrm{Y} \text { is true of } \mathrm{X} \text {, }
$$

\section{Because $\quad \mathrm{Z}$ is true of $\mathrm{X}$ (premise 1 ) \\ And $\quad \mathrm{Z}$ is symptomatic of $\mathrm{Y}$ (premise 2)}

In some circumstances, the arguer only expresses conclusions and premises 1 explicitly. Because in a specific context, the audience can usually "consciously complement" the premise 2 . To evaluate whether the argument scheme is properly used, we can raise three critical questions: (1) Is Z really a characteristic of Y? (2) Aren't there also other non Y's that have the characteristic Z? (3) Aren't there also other Y's that do not have the characteristic Z? (van Eemeren et al. 2002: 97-98)

The author uses the typical Symptomatic argument scheme in argument 1. Through discussion of the differences between the viewpoints and theories of Widdowson and Brumfit, the author intends 
to prove that the combination of Widdowson-Brumfit is inappropriate. The argument structure can be illustrated as :

Conclusion【be inappropriate】(Y) is true for【the conflation of Widdowson-Brumift】 (X)

Because【The viewpoints of Widdowson is different ...】(z) is true for【the conflation of Widdowson-Brumift】(X)(premise1)

And:【their theoris are different】(Z) is a characteristic of 【be inappropriate】 (Y) (premise 2)

From argument 1, we can conclude that evidence is not sound enough to support the reasoning process. Also, $\mathrm{Z}$ cannot be summarized as a characteristic of Y., resulting in a fallacy.

Secondly, argument 2 is also unreasonable and violates the rule of validness. In argument 2, the author contends that any reader can realize that Widdowson's work is more linguistic. However, he does not provide a set of rule to determine the extent of linguistics, thus violating the validity rule of argumentation.

\section{Conclusions}

\subsection{Major Findings}

In a pragma-dialectical view, we find that pragmatic argumentation shares a new perspective on the studies of academic argument. We identify and select pragmatic argumentation in the paper How Applied Linguistics is same as other science, according to the basic scheme of it and through reconstructing the argumentative discourse by identifying the standpoint and the argument, we find that it is usually readily supported by other types of argumentation so as to reasonably defend the standpoint better. Therefore, two types of pragmatic argumentation are put forward by combing pragmatic argumentation with two main types of argumentation divided by two main categories of argument schemes. They are pragmatic argumentation based on a symptomatic relation, and pragmatic argumentation based on causality. By categorizing pragmatic argumentation, we could identify them more easily in the academic argument and use them to enhance the effectiveness of persuasion in a reasonable way.

\subsection{Implication}

This analysis in this paper answers the questions in Section 3.2.1 and can be considered as a good example to identify and analyze complex pragmatic argumentation combing with other kinds of argumentation. Besides, this paper provides a new research domain about pragmatic argumentation. And studies on the academic argumentation help to provide a new set of rule in evaluation the reasonableness of it.

\subsection{Limitations}

This paper is trying to make a thorough analysis of one paragraph of an academic paper but there are still some limitations here:

The paragraph selected from the paper is not sufficient to make a systematic and complete analysis.

Due to the fact that the three types of argumentation respectively contain many subtypes, this paper is limited in terms of that only one specific subtype of the three types of argumentation is researched to combine with pragmatic argumentation.

The argument scheme of the two types of pragmatic argumentation is not summarized. 


\subsection{Future Research}

More academic papers can be selected in the future in order to make a thorough and scientific analysis of the academic argument. And pragmatic argumentation with all kinds of subtypes of the two types of argumentation in the paper should be involved. On the basis on that, the general argument scheme of two types of pragmatic argumentation should be given out if it is possible.

\section{References}

[1] Van Emerson, F.H, Identifying argumentative patterns: A vital step in the development of pragma-dialectics. Argumentation: this issue

[2] Van Emerson, F.H., Grootendorst, R.,\& Henkenmans, A.F.S.(2002). Argumentation: Analysis, evaluation, presentation. London: Routledge

[3] Yang Na, Wu Peng, 2016, A Study of Critical Thinking in the Perspective of Dialectical Discourse Analysis * Taking the teaching of Advanced Business English as an example, Foreign language community, 44-52

[4] Wu Peng, Lu Pin Chao, 2017, A Research of Pragmatic Argumentation on Media Diplomatic Discourse, Foreign language teaching, 55-60

[5] Chen Miao Yun, 1998, A Research On the Argumentation Method of Academic Papers, Journal of Hainan Normal University, No. 2, 7-12

[6] Xia Junjun, 2013, Argument, Argumentation and Critical Thinking in Academic Writing, Overseas English, 291-293

[7] Van Emerson, F.H, Xie Yu,2015 From the ideal model of "critical discussion" to the argumentative conversation in a specific context - the gradual development of the theory of argumentation of "pragmatic argumentation", Logic Research, 70-97 